\title{
The role of guide RNA structure in RNA interference
}

\section{Editorial}

Micro RNAs (miRNAs) and small interfering RNAs (siRNAs) represent the major triggers of RNA interference (RNAi) in most metazoan as well as in human cells. Two decades after first descriptions of the RNAi phenomenon, the RNAi pathway has been extensively studied. A common ground between miRNA- and siRNA-triggered RNAi is the formation of the RNA-induced silencing complex RISC, a multiprotein complex that includes a guide RNA which is either a processed mature miRNA or an antisense siRNA. ${ }^{1}$ During RNAi, guide RNAs direct RISC to complementary sites within target mRNAs leading either to translational repression or mRNA cleavage. The argonaute protein (Ago) as a core component of RISC engages a central role in the silencing pathway dividing the RNA guide into domains with distinct functions. ${ }^{2}$ The most important guide RNA domain is the hexamer or heptamer seed region (5' positions 2-7 or 2-8) which mediates the specificity of target binding of both miRNAs and siRNAs. The 5' terminal base is termed anchor and the regions following 3' of the seed are the central region (positions 9-12), the 3 ' supplementary region (positions 13-16), and the tail (positions 17-3'end). While siRNAs usually are fully complementary to their mRNA targets, miRNAs rarely pair extensively with their targets. However, for some miRNAs 3' supplementary pairing supports seed pairing.

In 2002 it was shown by Martinez et al., ${ }^{3}$ that siRNAs incorporated into RISC did not compete with a pool of free siRNAs anymore, suggesting that a RISC-loaded guide RNA is stably anchored in RISC and not interchangeable anymore. ${ }^{3}$ This hypothesis was supported by early structural studies on isolated domains of Argonaute proteins which identified conserved anchor sites for the 5' and 3' ends of the guide strand..$^{4-10}$ According to these studies, the guide strand 5' end is anchored in a basic pocket at the junction of the MID and PIWI domains and fixation of the 3 ' end occurs in the PAZ domain. Though positional base preferences were reported for antisense siRNAs and miRNAs on a statistical basis, Ago proteins can load small RNAs almost irrespective of sequence which is reflected in the absence of nearly any base-specific contacts. From the mechanistic point of view this makes sense since sequence constrains would confine the versatility of possible mRNA targets. The only exception is the 5, nucleotide of the guide strand, which is frequently uridine.

The fact that the guide RNA is part of a double-stranded precursor, either the duplex formed between a mature miRNA and its passenger strand or the siRNA duplex, prior to RISC formation and is then anchored at both ends after RISC loading, seemed to leave no room for guide RNA structure formation or any meaning of guide RNA structure in RNAi. On the other hand there was a lack of plausible explanations for the observation that, independent of the RISC loading potential of the guide RNA, some RISCs were found to be much more active than others. The reason for this could either rely on target-specific features or alternatively on the sequence or, perhaps, the structure of the guide RNA.

To address this question, possible correlations between the potential of antisense-siRNAs to fold secondary structures and
Volume I Issue 2 - 2014

Volker Patzel
Department of Microbiology, National University of Singapore,
Singapore

Correspondence: Volker Patzel, Department of Microbiology, Yong Loo Lin School of Medicine, National University of Singapore, Singapore I17597, Tel 6565163318, Fax 6567775720, Email micvp@nus.edu.sg

Received: May 12, 2014 | Published: May 13, 2014

the silencing activity of the corresponding siRNA duplexes were investigated..$^{11}$ For the sake of completeness, target structures were described to impact RNAi as well [reviewed in], ${ }^{12}$ however, by the design of this study, both guide RNA sequence- and target sequence or structure-related influences could be excluded. In addition, any influence of RNA secondary structure folding on the quality of the tested siRNA duplexes was excluded. The results demonstrated a strong inverse correlation between the potential of guide RNAs to fold stable secondary structures and siRNA-mediated gene silencing. Unstructured guide strands were found to be most active whereas stem-loop structures with base-paired ends were inactive. ${ }^{11}$ Thus, this analysis pointed towards a role of guide RNA structure formation in siRNA-mediated RNAi. Despite this clear correlation and its predictive power for functional siRNA design, the questions how these observations could match with the mechanistic model of RNAi and at which stage of the silencing pathway guide structure might become relevant, remained unanswered.

Only more recent crystal structures of binary and ternary complexes of Ago proteins, mainly of the Thermus thermophilus argonaute (TtAgo), harbouring either the guide strand alone or both guide and target strands, leave room for new plausible interpretations. ${ }^{13-16}$ The structures of the binary complexes displayed the guide sequence anchored at both ends and the Watson-Crick edges of seed bases in a near A-helical conformation positioned for target nucleation. Together, the structures of binary and ternary complexes support an earlier suggested dynamic two-state model of guide end tethering and Ago function: ${ }^{17}$ While the 5 ' end of the guide always remains fixed, the 3 ' end is anchored in the PAZ pocket in the binary complex constituting a cleavage-incompatible conformation, and is released in the ternary complex yielding a cleavage-compatible conformation. ${ }^{18}$ However, 3 ' end release was only observed for guide strands of 15 nucleotides or longer forming at least 14 base pairs (bps) with the target while no 3' end release was observed for shorter guides or guides forming less bps, indicating that a propagating duplex of a distinct minimum length that typically includes the 3' supplementary region, generates sufficient binding energy to tear out the 3 ' end from the PAZ pocket.

While 3' end release represents a prerequisite for the formation 
of a cleavage-competent RISC on the one hand, it basically enables refolding of structure-competent guide strands that could compete with target binding and RNA silencing on the other hand. Thus, during siRNA-triggered RNAi, guide structure formation may come into play at two topologically analogous stages: firstly, in the preRISC after duplex loading and upon passenger strand cleavage and release, and secondly, upon nucleation and duplex propagation of the target-bound mature RISC. In both situations, refolding of the 5' anchored, 3' released guide strand may lead to silencing-incompetent RISCs. Notably, no 3' end release was observed in a ternary complex resembling a miRNA-like TtAgo silencing complex, i.e. an Ago silencing complex harbouring a target strand that mismatches to the guide positions 10 and $11 .{ }^{13}$ Given the very limited data available on miRNA-like silencing complexes, no final conclusion as to whether guide structure formation plays a role in miRNA-triggered RNAi or not can presently be made. However, extensive computational secondary structure analyses of mature miRNAs and supplementary experiments, point towards a role of guide RNA structure in miRNAtriggered RNAi (Patzel et al., yet to publish). ${ }^{12}$

In conclusion, recent data on the crystal structures of binary and ternary Ago silencing complexes do no longer stand at odds with observed correlations between guide RNA secondary structure formation and RNAi and a model in which guide structure formation may compete with target binding of RISC and, thus, negatively interfere with RNA silencing.

\section{Acknowledgements}

This work was supported by the NUS-Cambridge Start-up grant of the National University of Singapore (grant number R-182-000163-646); the National Medical Research Council of Singapore (grant number NMRC/NIG/1058/2011) and the Academic Research Fund of the Ministry of Education of Singapore (grant number R-182-000200-112).

\section{Conflict of interest}

Author declares that there is no conflict of interest.

\section{References}

1. Kawamata T, Tomari Y. Making RISC. Trends Biochem Sci. 2010;35(7):368-376.

2. Wee LM, Flores-Jasso CF, Salomon WE, et al. Argonaute divides its RNA guide into domains with distinct functions and RNA-binding properties. Cell. 2012;151(5):1055-1067.
3. Martinez J, Patkaniowska A, Urlaub H, et al. Single-stranded antisense siRNAs guide target RNA cleavage in RNAi. Cell. 2002;110(5):563-574.

4. Lingel A, Simon B, Izaurralde E, et al. Structure and nucleicacid binding of the Drosophila Argonaute 2 PAZ domain. Nature. 2003;426(6965):465-469.

5. Yan KS, Yan S, Farooq A, et al. Structure and conserved RNA binding of the PAZ domain. Nature. 2003;426(6965):468-474.

6. Song JJ, Liu J, Tolia NH, et al. The crystal structure of the Argonaute2 PAZ domain reveals an RNA binding motif in RNAi effector complexes. Nat Struct Biol. 2003;10(12):1026-1032.

7. Ma JB, Ye K, Patel DJ. Structural basis for overhang-specific small interfering RNA recognition by the PAZ domain. Nature. 2004;429(6989):318-322.

8. Lingel A, Simon B, Izaurralde E, et al. Nucleic acid 3'-end recognition by the Argonaute2 PAZ domain. Nat Struct Mol Biol. 2004;11(6):576-577.

9. Ma JB, Yuan YR, Meister G, et al. Structural basis for 5'-end-specific recognition of guide RNA by the A. fulgidus Piwi protein. Nature. 2005;434(7033):666-670.

10. Parker JS, Roe SM, Barford D. Structural insights into mRNA recognition from a PIWI domain-siRNA guide complex. Nature. 2005;434(7033):663-666.

11. Patzel V, Rutz S, Dietrich I, et al. Design of siRNAs producing unstructured guide-RNAs results in improved RNA interference efficiency. Nat Biotechnol. 2005;23(11):1440-1444.

12. Patzel V. In silico selection of active siRNA. Drug Discovery Today. 2007;12(3-4):139-148.

13. Wang Y, Juranek S, Li H, et al. Structure of an Argonaute silencing complex with a seed-containing guide DNA and target RNA duplex. Nature. 2008;456(7224):921-926.

14. Wang Y, Sheng G, Juranek S, et al. structure of the guide-strand-containing argonaute silencing complex. Nature. 2008;456(7219):209-213.

15. Wang $\mathrm{Y}$, Juranek $\mathrm{S}$, Li $\mathrm{H}$, et al. Nucleation, propagation and cleavage of target RNAs in Ago silencing complexes. Nature. 2009;461(7265):754-761.

16. Schirle NT, MacRae IJ. The crystal structure of the human Argonaute2. Science. 2012;336(6084):1037-1040.

17. Tomari Y, Zamore PD. Perspective: machines for RNAi. Genes Dev. 2005;19(5):517-529.

18. Sheng G, Zhao H, Wang J, et al. Structure-based cleavage mechanism of Thermus thermophilus Argonaute DNA guide strand-mediated DNA target cleavage. Proc Natl Acad Sci U S A. 2013;111(2):652-657. 